\title{
DATA DRIVEN FEATURE IDENTIFICATION AND SPARSE REPRESENTATION OF TURBULENT FLOWS
}

\author{
Mohammad Beit-Sadi \\ Department of Aeronautics \\ Imperial College London \\ London \\ mb4413@imperial.ac.uk
}

\author{
Jakub Krol \\ Department of Aeronautics \\ Imperial College London \\ London \\ jakub.krol09@imperial.ac.uk
}

\author{
Andrew Wynn \\ Department of Aeronautics \\ Imperial College London \\ London \\ a.wynn@imperial.ac.uk
}

\begin{abstract}
Identifying coherent structures of fluid flows is of great importance for reduced order modelling and flow control. Finding such structures in a turbulent flow, however, can be challenging. A number of modal decomposition algorithms have been proposed in recent years which decompose snapshots of data into spatial modes, each associated with a single frequency and growth-rate, most prominently dynamic mode decomposition (DMD). However, the number of modes that DMD-like algorithms construct may be unrelated to the number of significant degrees of freedom of the underlying system. This provides a difficulty if one wants to create a low-order model of a flow. In this work, we present a method of post-processing DMD modes for extracting a small number of dynamically relevant modes. This is achieved by first ranking the DMD modes, then using an iterative approach based on the graph-theoretic notion of maximal cliques to identify clusters of modes and, finally, by replacing each cluster with a single (pair of) modes.
\end{abstract}

\section{Introduction}

A number of pure-frequency modal decomposition algorithms have been developed in recent years. Most notably, dynamic mode decomposition (DMD) proposed by Schmid (2010), which uses an ensemble of time-resolved snapshots to find spatial modes, each of which is associated with a single frequency and growth rate.

In particular, given an ensemble $\mathscr{X}=\left\{x_{i}\right\}_{i=1}^{N+1} \subset \mathbb{R}^{p}$ of $N+1$ snapshots sampled at a common timestep $\Delta t$, consider matrices

$$
X=\left(\begin{array}{cccc}
\uparrow & \uparrow & & \uparrow \\
x_{1} & x_{2} & \cdots & x_{N} \\
\downarrow & \downarrow & & \downarrow
\end{array}\right), \quad X^{\prime}=\left(\begin{array}{cccc}
\uparrow & \uparrow & & \uparrow \\
x_{2} & x_{3} & \cdots & x_{N+1} \\
\downarrow & \downarrow & & \downarrow
\end{array}\right)
$$

DMD approximates the evolution of the data observed in the ensemble by constructing a model of the form

$$
x_{i+1} \approx U \tilde{A} U^{\top} x_{i}
$$

over the sampling timestep $\Delta t$. Here, $U \in \mathbb{R}^{p \times N}$ is taken from the singular value decomposition $X=U \Sigma V^{\top}$, while $\tilde{A} \in \mathbb{R}^{N \times N}$ is the solution of the optimization problem

$$
\tilde{A}=U^{\top} X^{\prime} V \Sigma^{-1}=\operatorname{argmin}_{A}\left\|X^{\prime}-U A U^{\top} X\right\|_{F}^{2},
$$

where $\|\cdot\|_{F}$ denotes the Frobenius norm.

Now, let $\tilde{A}=P \Lambda P^{-1}$ be an eigendecomposition of $\tilde{A}$ with eigenvectors $p_{i}$ and eigenvalues contained in $\Lambda=$ $\operatorname{diag}\left(\lambda_{i}\right)$. Since $U \tilde{A} U^{\top}$ approximates the observed evolution over a timestep $\Delta t$, the DMD modes $\phi_{i}$ are defined by $\phi_{i}=U p_{i}$, each of which is associated with a DMD eigenvalue $\mu_{i}=\log \left(\lambda_{i}\right) / \Delta t$.

In its standard implementation, DMD obtains as many modes as the number of snapshots. Typically, one would like to determine which of these have the most significant contribution to the underlying dynamics. One way to do this is to appeal to the Koopman approximation

$$
x_{j} \approx \sum_{i=1}^{N} \alpha_{i} \phi_{i} \lambda_{i}^{j-1},
$$

where $\phi_{i} \in \mathbb{C}^{p}$ are the DMD modes, normalised so that $\left\|\phi_{i}\right\|_{2}=1$, and $\alpha_{i} \in \mathbb{C}$ are constants. For sequential data, these are usually obtained by substituting $j=1$ in 3 and solving the linear least-squares problem

$$
\min _{\left(\alpha_{i}\right)_{i=1}^{n}}\left\|x_{1}-\alpha^{\top} \Phi\right\|_{F}^{2}
$$

where $\alpha$ is the column vector containing the constants $\alpha_{i}$. This, and a similar ranking method which is more suitable for non-sequential data were proposed by Tu et al. (2013) as methods of scaling the modes according to the significance of their contribution to the data. The value of $\left|\alpha_{i}\right|$ is referred to as the amplitude of the mode $\phi_{i}$ and is used as a proxy for the its significance (see Schmid (2011) and Grilli et al. (2012) for examples), suggesting that a sparse representation of the flow could be built using modes with the largest amplitudes.

However, Hemati et al. (2017) shows that the classical implementation of DMD essentially assumes that all the noise is contained in the time-shifted snapshots $X^{\prime}$ and that the prior snapshots $X$ are uncorrupted. As a result, the DMD can over-fit in order to accommodate for the noise in the original snapshots, and the author's propose the Total DMD method to attempt to address this problem. In this paper, we observe a similar phenomenon when DMD is applied to PIV snapshots of a turbulent bluff body flow at high Reynolds number, where highly damped (i.e. $\operatorname{Re}\left(\mu_{i}\right) \ll 0$ ), spatially incoherent modes are nonetheless highly-ranked in the amplitude ordering suggested by (4).

An alternative way of finding a more sparse model for the system is to calculate a small number of modes. The simplest way of achieving this aim is to replace $U$ in 2 by 
the truncation $U_{r} \in \mathbb{R}^{p \times r}$ whose columns are only the first $r$ singular vectors of $X$, and replacing $\Sigma$ and $V$ by analogously truncated matrices and forming $\tilde{A}_{r}=U_{r}^{\top} X^{\prime} V_{r} \Sigma_{r}^{-1}$. In this case only $r$ modes

$$
\left\{\phi_{i}\right\}_{i=1}^{r}=: D M D(\mathscr{X}, r)
$$

are obtained in an analogous manner to DMD, using the eigendecompsition of $\tilde{A}$. A similar approach is taken by the optimal mode decomposition (OMD) algorithm. In this case, a projection matrix $L \in \mathbb{R}^{p \times r}$ of user-defined rank $r$ is considered and the optimization problem (2) solved with $U$ replaced by $L$, and with both $L$ and $A \in \mathbb{R}^{r \times r}$ as optimization variables. Again, this approach produces a potentially small number of, hopefully, dynamically relevant modes.

A question still remains, however, as to which value of the truncation dimension $r$ to select. One could implement a range of values of $r$ and analyse the sensitivity of the resulting decomposition, an approach followed by Baj et al. (2015) for flow past in the near wake of a multiscale array. However, this such a process may be lengthy. Furthermore, dramatically reducing the truncation dimension can have the effect of modifying the spectral information associated with each mode. Figure 1 shows that the discrete-time eigenvalues $\lambda_{i}$ for a typical (specifically, the bluff body flow considered in 3 DMD implementation and indicates that that mode damping ratio is decreased as $r$ decreases.

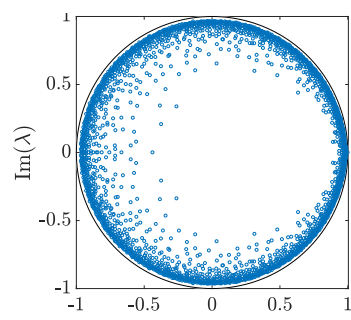

(a)

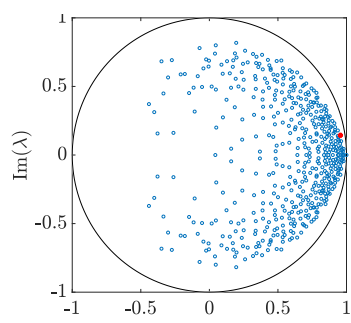

(c)

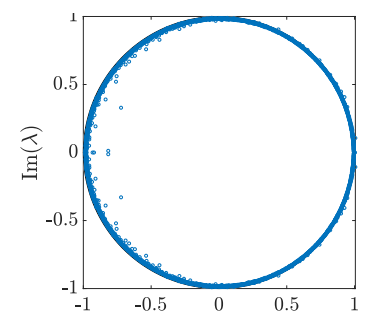

(b)

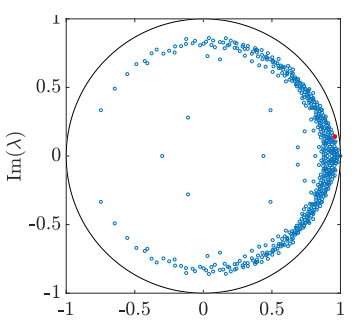

(d)
Figure 1. Discrete-time eigenvalues of DMD when applied to the data discussed in $\$ 3$ at a) $r=2500, b) r=500$, and OMD eigenvalues at b) $r=2500$ and d) $r=500$.

In other words, the benefit of constructing only a small number comes at the cost of potentially corrupted spectral information. Consequently, an alternative approach is to first compute a large number of modes, then post-process the result to either pick or generate a smaller number of representative modes. Sparsity promoting DMD (DMDsp), proposed by Jovanovic et al. (2014), achieves this by solving the optimisation problem

$$
\underset{\alpha}{\operatorname{minimize}}\left\|X-\Phi D_{\alpha} \hat{V}\right\|_{F}^{2}+\gamma\|\alpha\|_{1},
$$

where $X$ is the matrix of the snapshots, $\Phi$ is a matrix whose columns are the normalised DMD modes, $D_{\alpha}:=\operatorname{diag}\left(\alpha_{i}\right)$ and $\hat{V}:=\left(\lambda_{i}^{j-1}\right)_{i, j=1}^{n}$ is the Vandemonde matrix formed from the DMD eigenvalues. Finally, $\gamma \geq 0$ is a parameter which encourages a sparse solution to the optimization problem. The first term in (5) amounts to fixing $\lambda_{i}, \Phi_{i}$ and finding the optimal coefficients $\alpha_{i}$ in 3 , while the second seeks to penalise a large number of non-zero values of $\alpha_{i}$.

DMDsp, however, does not calculate new modes or eigenvalues. Instead, it retains a sparse subset of the original DMD modes. The $\ell_{1}$-norm regularisation allows the user to specify the emphasis on the sparsity of the model, and thus models of various orders can be constructed. Further sensitivity analysis is therefore necessary to determine a sensible number of modes to use for reduced order modelling.

In this paper, we propose an alternative method which seeks to reduce the order of the model by analysing the spatial similarity of modes. It first identifies sets of significant modes, and then refines the original modes. The method identifies clusters of spatially similar modes, and replaces the modes in those cluster, with a single conjugate pair of modes.

\section{Methodology}

Figure 1 suggest that, when a large number of modes $r \gg 1$ are constructed, modal decomposition algorithms such as DMD may produce multiple modes with similar spectral information and, correspondingly, similar spatial features. These similarities can directly exploited to obtain a sparse representation of the underlying system. To do so, however, requires a definition of mode similarity.

Assuming that the underlying data ensemble is realvalued, DMD modes and eigenvalues are either themselves real or come in conjugate pairs. As a consequence, the Koopman decomposition (3) of the underlying data ensemble implies that the proportion of the data described by the model (1), that is $U U^{\top} \mathscr{X}$, satisfies

$$
U U^{\top} \mathscr{X} \subseteq \bigcup_{i=1}^{n} \operatorname{span}_{\mathbb{R}}\left(\operatorname{Re}\left(\phi_{i}\right), \operatorname{Im}\left(\phi_{i}\right)\right)
$$

Consequently, a possible interpretation of contribution of the DMD mode $\phi_{i}$ to the underlying data ensemble is that subspace spanned by its real and imaginary parts. This suggests a possible measure of mode similarity.

Given a DMD mode $\phi_{i} \in \mathbb{C}^{p}$, let $A_{i}:=$ $\left[\operatorname{Re}\left(\phi_{i}\right), \operatorname{Im}\left(\phi_{i}\right)\right] \in \mathbb{R}^{p \times 2}$. For modes $\phi_{i}$ and $\phi_{j}$, the statistic

$$
\theta_{i j}=\sin ^{-1}\left(\left\|A_{i}-A_{j}\left(A_{i}^{\top} A_{j}\right)\right\|_{2}\right)
$$

where $\|\cdot\|_{2}$ denotes the largest singular value of a matrix, is the smallest angle between the subspaces spanned by their respective real and imaginary parts, described by Bjorck \& Golub (1973). For simplicity, we consider $\cos \left(\theta_{i j}\right) \in[0,1]$ as the measure of mode similarity, with 0 denoting the least similar modes, and 1 for modes which span the same subspace of $\mathbb{R}^{p}$.

Our aim is to find clusters of spatially similar modes. To achieve this, will will draw upon techniques from graph theory. In the following, it will be useful to express the similarity pattern of a set of modes in a binary matrix. In 
particular, given a set $\left\{\phi_{i}\right\}_{i=1}^{n}$ of modes and a tolerance level $0 \leq \varepsilon \leq 1$, define coefficients $d_{i j} \in\{0,1\}$ by

$$
d_{i j}=\left\{\begin{array}{lll}
1, & \text { if } & \cos \left(\theta_{i j}\right)-\delta_{i j} \geq \varepsilon, \\
0, & \text { if } \quad & \cos \left(\theta_{i j}\right)-\delta_{i j}<\varepsilon .
\end{array}\right.
$$

We write $d\left(\left\{\phi_{i}\right\}_{i=1}^{n}\right):=\left(d_{i j}\right)_{i, j=1}^{n} \in \mathbb{S}^{n}$ as the symmetric matrix created by these coefficients, with the interpretation that a pair of modes are spatially similar if $d_{i j}=1$ and dissimilar otherwise if $d_{i j}=0$. Note that $d_{i i}=0$, meaning that we discount a mode's inherent self-similarity.

\subsection{A Graph Theoretical Approach}

A graph $\mathscr{G}=(\mathscr{V}, \mathscr{E})$ consists of a set of vertices $\mathscr{V} \subset \mathbb{N}$ and a set of edges $\mathscr{E} \subseteq \mathscr{V} \times \mathscr{V}$, where $(i, j) \in \mathscr{E}$ implies that there is an edge connecting vertex $i$ to vertex $j$. A graph is called undirected when $(i, j) \in \mathscr{E}$ if and only if $(j, i) \in \mathscr{E}$. If $\mathscr{V}=\{1,2, \ldots, n\}$, the edge pattern of an undirected graph can be represented by a symmetric adjacency matrix $A=$ $\left(a_{i j}\right) \in \mathbb{S}^{n}$ with $a_{i j}=1$ if $(i, j) \in \mathscr{E}$ and $a_{i j}=0$ otherwise. In this case, we write $(\mathscr{V}, \mathscr{E})=(\mathscr{V}, A)$.

Now, given a set $\left\{\phi_{i}\right\}_{i=1}^{n}$ of DMD modes, we may associate with them an undirected graph $\left(\{1,2, \ldots, n\}, d\left(\left\{\phi_{i}\right\}_{i=1}^{n}\right)\right)$. The problem of identifying clusters of modes is now re-expressed as one of finding subsets of connected vertices. In this context, a useful graph-theoretic notion is that of a clique which, by definition, is a complete subgraph (i.e., one where each pair of vertices are connected by an edge). A maximal clique is a clique which is not strictly contained in any other clique.

Maximal cliques can be obtained using the BronKerbosch algorithm Bron \& Kerbosch (1973), although in this paper we employ a more computationally-efficient variant of Tomita et al. (2006), which bounds the number of calculations by $O\left(3^{n / 3}\right)$, where $n$ is the number of graph vertices. Using maximal cliques to group modes ensures that as many similar vertices as possible are contained within each cluster and that the resulting decomposition is, in a sense, minimal. In the following, given a graph $\mathscr{G}=(\mathscr{V}, A)$, we let $c(\mathscr{G}) \subseteq 2^{\mathscr{V}}$ denote the set of maximal cliques (which is a set of subsets of $\mathscr{V}$ ).

We now describe an iterative algorithm, using the notion of maximal cliques, that may be used to identify a small number of modes of interest from a large initial ensemble. As above, we begin with a set of DMD modes $\left\{\phi_{i}\right\}_{i=1}^{n}$ and define, initially, the coarsest-possible set of clusters $C_{j}^{(0)}:=\left\{\phi_{j}\right\}$, for each $j=1, \ldots, n$. Next, consider the graph $\mathscr{G}_{0}:=\left(\{1,2, \ldots, n\}, D_{0}\right)$ with adjacency matrix $D_{0}=d\left(\left\{\phi_{i}\right\}_{i=1}^{n}\right)$ and let $\left\{H_{j}^{(1)}\right\}_{j=1}^{n_{1}}=c\left(\mathscr{G}_{0}\right)$ be the maximal cliques. For each $j=1, \ldots, n_{1}$, we call

$$
C_{j}^{(1)}:=\left\{\phi_{i}: i \in H_{j}^{(1)}\right\}=\bigcup_{i \in H_{j}^{(1)}} C_{i}^{(0)}
$$

the cluster formed of the underlying modes associated with the clique $H_{j}^{(1)}$.

We now seek to construct a representative mode for each cluster. To do this, for each $j=1, \ldots, n_{1}$, let $P_{j}^{(1)} \in$ $\mathbb{R}^{p \times p}$ be the orthogonal projections onto

$$
\bigcup_{i \in H_{j}^{(1)}} \operatorname{span}_{\mathbb{R}}\left(\operatorname{Re}\left(\phi_{i}\right), \operatorname{Im}\left(\Phi_{i}\right)\right) \subset \mathbb{R}^{p}
$$

Next, define the projection of the full snapshot ensemble $\mathscr{X}$ onto the modes of each clique by $\mathscr{X}_{j}^{(1)}:=P_{j}^{(1)} \mathscr{X}$. Since each $\mathscr{X}_{j}$ belongs to a subspace of $\mathbb{R}^{p}$ spanned by spatially similar modes, we seek to find a single average representative mode by solving $\left.\left\{\phi_{j}^{(1)}\right\}:=D M D\left(\mathscr{X}_{j}^{(1)}, 3\right)\right]^{1}$ to obtain a representative mode of the clique $H_{j}^{(1)}$.

The subsequent iteration is to form cliques of the representative modes. To do this, create the adjacency matrix $D_{1}:=d\left(\left\{\phi_{j}^{(1)}\right\}_{j=1}^{n_{1}}\right)$, graph $\mathscr{G}_{1}=\left(\left\{1, \ldots, n_{1}\right\}, D_{1}\right)$ and maximal cliques $\left\{H_{j}^{(2)}\right\}_{j=1}^{n_{2}}=c\left(\mathscr{G}_{1}\right)$. The second-generation clusters of modes are then defined by

$$
C_{j}^{(2)}:=\bigcup_{i \in H_{j}^{(2)}} C_{i}^{(1)}, \quad j=1, \ldots, n_{2} .
$$

This process can be repeated iteratively, detailed in Algorithm 1 by forming representative modes for each new cluster, forming the associated undirected graph and then obtaining its maximal cliques. Since we are not considering the self-similarity of the modes, once all the similar clusters have coalesced and the algorithm cannot find any similarity between the representative modes, the algorithm terminates.

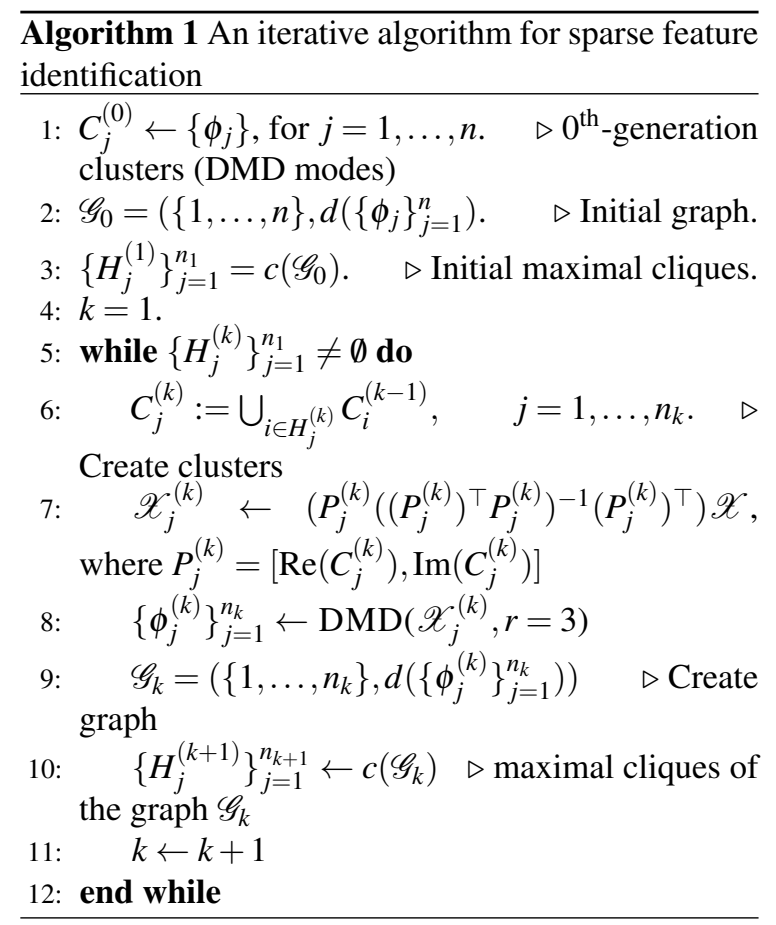

In line 8 of Algorithm 1, one could of course substitute any modal decomposition algorithm which is able to generate a specified number of modes.

At a given iteration $k$, the algorithm regroups the original DMD modes into different clusters, through the similarity of the representative modes $\phi_{j}^{(k)}$. It is important to note that at a given iteration, one or more representative mode may not be similar to any others. Consequently, the clusters represented by such modes will appear in the subsequent clusters $C_{j}^{(k+1)}$. This does not imply that $\phi_{s}^{(k)}$ should now

\footnotetext{
${ }^{1}$ Running the decomposition at $r=3$ results in one complex conjugate pair of modes one real-valued mode. We keep the complex mode only.
} 
be disregarded, merely that the flow features represented by it are judged distinct from those described by the remaining representative modes. Consequently, we require a measure of cluster significance which can be applied irrespective of the algorithm iteration.

\subsection{Ranking Clusters}

We describe a measure of significance for both individual modes and the identified clusters $C_{j}^{(k)}$. To do this, first find coefficients $\alpha_{i}$ by solving the optimisation problem

$$
\min _{\left(\alpha_{i}\right)_{i=1}^{n}}\left\|X-\Phi D_{\alpha} \hat{V}\right\|_{F}^{2}
$$

where $\Phi$ is the matrix whose columns are the normalised DMD modes, $D_{\alpha}:=\operatorname{diag}\left(\alpha_{i}\right)$ and $\hat{V}:=\left(\lambda_{i}^{j-1}\right)_{i, j=1}^{n}$ is the Vandemonde matrix formed using the DMD eigenvalues. This is equivalent to solving (5) with $\gamma=0$.

On their own, these values may give an inaccurate representation of the significant features in the flow, since a mode may have a large constant $\alpha_{i}$ yet a small associated eigenvalue $\left|\lambda_{i}\right| \ll 1$. For this reason, we define the measure of significance for each mode by

$$
\sigma\left(\phi_{i}\right):=\left\|\alpha_{i} v_{i}\right\|_{2}
$$

where $\mathbf{v}_{\mathbf{i}}$ is the $i t h$ row of the Vandemonde matrix $\hat{V}$. The significance of a cluster defined by

$$
\sigma\left(C_{j}\right):=\sum_{\left\{i: \phi_{i} \in C_{j}\right\}} \sigma\left(\phi_{i}\right)
$$

If two clusters join to form a larger cluster in the next generation, i.e. if $C_{i}^{(k)}, C_{j}^{(k)} \subset C_{\ell}^{(k+1)}$, then the contributions of all the modes which make up the smaller cluster are accounted for in $\sigma\left(C_{\ell}^{(k+1)}\right)$. This will make $C_{i}^{(k)}$ and $C_{j}^{(k)}$ redundant. However, if $C_{i}^{(k)}$ is not contained in any cluster formed during the next iteration, then $\sigma\left(C_{i}^{(k)}\right)$ can be used for comparison with other clusters, including the ones created during the later iterations.

\section{Results}

We consider flow past an axisymmetric bluff body at $\operatorname{Re}=1.88 \times 10^{5}$, the experimental setup of which is detailed by Oxlade et al. (2015). The free-stream velocity is $U_{\infty}=15 \mathrm{~ms}^{-1}$, base diameter $D=0.1965 \mathrm{~m}$, with a length to diameter ratio of $L / D=6.8$. Before analysing these results and extracting clusters of modes using Algorithm 1 . we review the known spatio-temporal features of similar axisymmetric bluff-body flows.

Berger et al. (1990) analysed experimental hot-wire data from flows past a circular disc for $1.5 \times 10^{4} \leq R e \leq$ $3 \times 10^{5}$, and observed that the farthest point of the recirculation bubble from the base of the disc oscillates at $S t \approx 0.05$ (throughout, $S t$ is the Strohual number based on body diameter and free-stream velocity) about its mean streamwise location. They dubbed this extension and contraction of the recirculation region, the bubble-pumping mode. They also observed a high-frequency peak at $S t \approx 1.62$ in the power spectrum of the velocity time series sampled in the immediate vicinity of the separated shear layer.

More recently, Rigas et al. (2014) used a POD of the base pressure data from the same experimental setup considered in this paper, at $\operatorname{Re}=1.88 \times 10^{5}$. They observed an axisymmetric base-pressure structure associated with bubble-pumping at $S t \approx 0.05$, an asymmetric structure associated with vortex shedding at $S t \approx 0.2$, and the random rotation of the vorticity lobes as the dominant feature of the flow. Both Berger et al. (1990) and Rigas et al. (2014) explain the flow to be asymmetric in the rotational frame of reference, with axisymmetry only achieved in an average sense. Both show that the boundary of the recirculation region oscillates about its mean position at $S t_{D} \approx 0.05$. However, the localised high frequency feature at $S t \approx 1.6$ associated with the shear-layer instability were not observed in the base-pressure data considered by Rigas et al. (2014).

For the current study, we compute DMD modes of a continuous sample of 2732 snapshots $\mathscr{X}$, sampled at 720 $\mathrm{Hz}$, of the velocity field up to $1.72 \mathrm{D}$ downstream of the body's base. The data ensemble consists of flow fields where, on average, the azimuthal orientation of the wake's plane of asymmetry as approximated using the position of the centre of pressure, were approximately perpendicular to the PIV plane of view. Although the variance of this azimuthal position is $\frac{\pi}{5}$, most of the variation is concentrated at two short intervals, each lasting about $0.1 \mathrm{~s}$ (comprising approximately $5 \%$ of the data). Since the field of view is approximately perpendicular to the plane of asymmetry, both axisymmetric and antisymmetric features will be detectable. As explained above, this includes the vortex shedding, shear layer instability and bubble pumping modes. Figure 2 (a) shows the streamwise component of the mode associated with the mean velocity field.

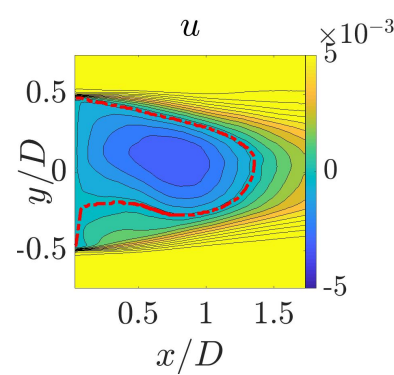

(a)

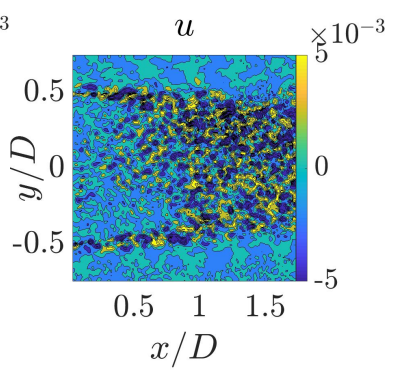

(b)
Figure 2. Mode associated with the mean flow (a) and the real part of the DMD mode at $S t \approx 2.9$ (b). The average position of the recirculation region is marked by the red dotted line.

DMD modes were computed using $r=2731$ and ranked using, first, the classical ranking method (4) and, second, the method of 2.2 Results of the classical ranking are shown in Figure 3 Surprisingly, the second-ranked mode at $S t \approx 2.9$, which is highly damped $\left(\operatorname{Re}\left(\mu_{i}\right)=-84\right)$ and spatially incoherent, as shown in Figure 2 (b), is ranked higher than the dynamically important modes corresponding to shedding and bubble-pumping. Contrastingly, Figure 4 shows the DMD mode ranking computed using (7), which serves to emphasise less damped modes of increased 
spatial coherence. For improved legibility, the mode associated with the mean flow is not included in this figure.

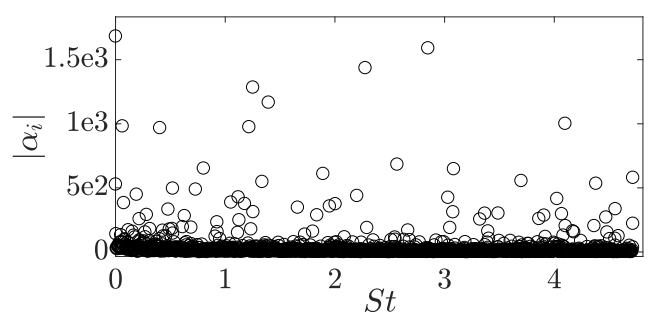

Figure 3. Classic ranking of the of the DMD modes' energetic contribution, $\left|\alpha_{i}\right|$, using (4), against decomposition Strouhal number.

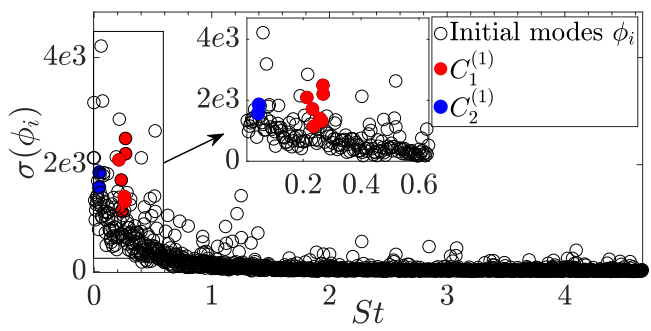

Figure 4. Energetic contribution of the DMD modes (hollow circles) against Strouhal number. The constituent modes of clusters $C_{1}^{(1)}$ (red) and $C_{2}^{(1)}$ (blue).

We now apply Algorithm 1 to create clusters of DMD modes. Algorithm 1 is applied with a cut-off tolerance of $\varepsilon=0.5$, using DMD for the first three iterations and the OMD algorithm (in line 8 on Algorithm 1) in subsequent iterations. The motivation for this choice is that DMD is computationally efficient, while OMD produces extracts more accurate spatio-temporal features, which is of importance for later iterations. To further reduce computational cost, clusters with whose modes have a mean Strouhal number, written $\langle S t\rangle$, greater than 2.5 are omitted from subsequent iterations of the algorithm. With these settings, Algorithm 1 terminates after five iterations.

Although the clusters are constructed by considering the spatial features of a mode, the two clusters highlighted in Figure 4, which are both first generation clusters, illustrate that (for this dataset) modes within clusters typically possess similar spectral information.

Figure 5 shows the aggregate energetic contribution of the clusters at each iteration of the algorithm, computed using (8) plotted against the mean decomposition frequency associated with that cluster. For each of the first three iterations, the (locally) highly-ranked clusters have mean frequencies in four distinct bands $\langle S t\rangle \approx 0.01-0.07,0.15-0.25$, $0.4-0.5$ and $1.2-1.25$. It is interesting to contrast this with the more widely-dispersed frequency distribution of the original DMD in Figure 4 Returning to Figure 5 the clusters at $\langle S t\rangle \approx 0.15-0.25$ coalesce with those at $\langle S t\rangle \approx 0.4-0.5$ during the fourth iteration, rendering the third generation clusters at $\langle S t\rangle \approx 0.4-0.5$ redundant.
Once all the redundant clusters are removed, the remaining clusters can then be compared using their ranks $\sigma\left(C_{j}\right)$. The inset plot in Figure 5 shows the clusters that remain after termination of Algorithm 1 The inset indicates that Algorithm 1 obtains a sparse representation of system where two clusters, $C_{1}^{(5)}$ and $C_{2}^{(5)}$ and, hence, their representative modes, are nearly two degrees of magnitude more significant compared to the other modes. Though considerably less energetic, the third cluster is one generated during the third iteration and has $\langle S t\rangle \approx 1.2$. The representative mode contains features with small length scales. Though the significance of this mode must be further analysed, it appears to be coherent in the vicinity of the shear layer, and spatially incoherent otherwise.

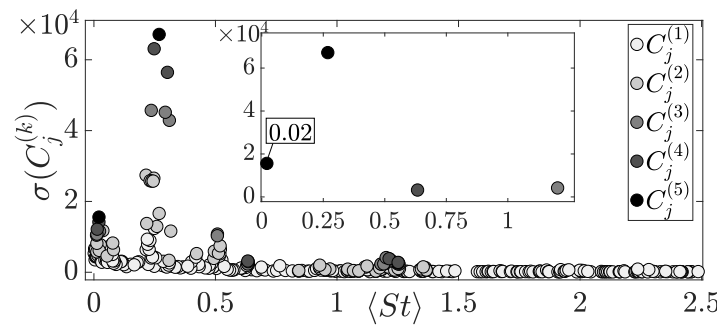

Figure 5. Aggregate ranking of the clusters in $1^{\text {st }}$ to $5^{\text {th }}$ generation clusters, against the mean decomposition Strouhal number of their constituent modes.

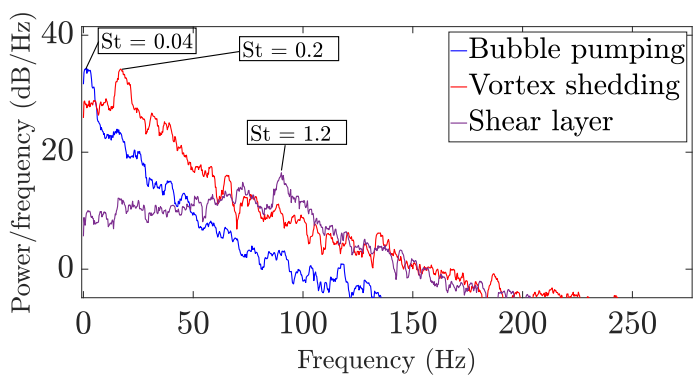

Figure 6. Power spectral density of the temporal coefficients of the post processed modes.

The streamwise velocity component of the the representative modes of the three identified clusters are illustrated in Figure 7 Consistent with obervations of both Rigas et al. (2014) and Berger et al. (1990), the highestranked modes correspond to vortex shedding and bubble pumping which are the dominant features of the flow in the rotating frame of reference.

Figure 7 (a) and (b) present the real and imaginary parts of the mode from the dominant cluster. This represents periodic vortex shedding, predominant in the region $y>0$, as a result of the slight assymetry of the flow apparant in figure 2 (a). The representative mode for the cluster with $\langle S t\rangle \approx 0.04$ is shown in Figure 7 (c) and (d). This represents the bubble pumping mode, with relative weights of the real and imaginary parts able to produce average movement of the recirculation region in both streamwise and $y$ directions. Finally, as observed above, the representative mode shown 


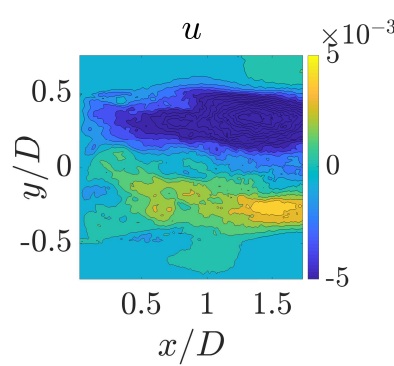

(a)

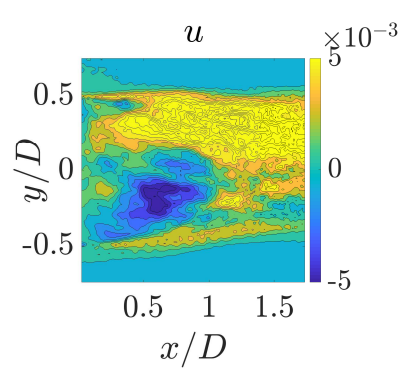

(c)

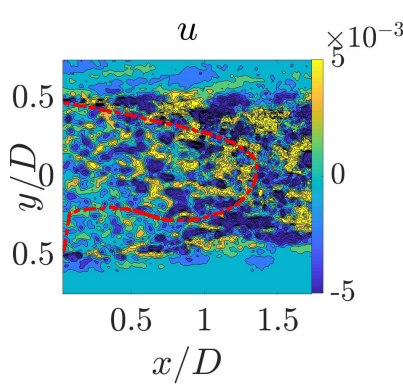

(e)

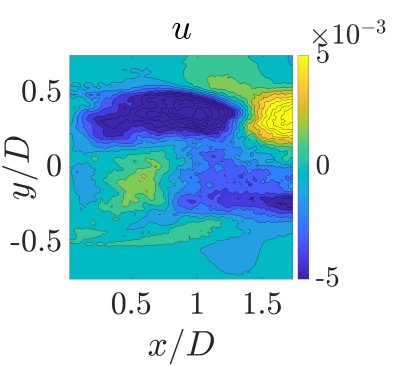

(b)

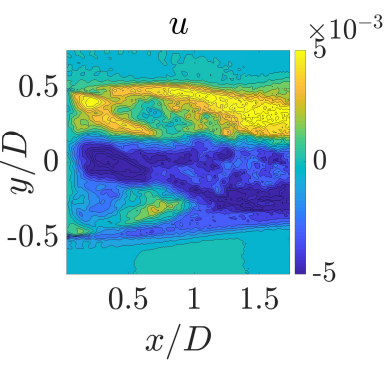

(d)

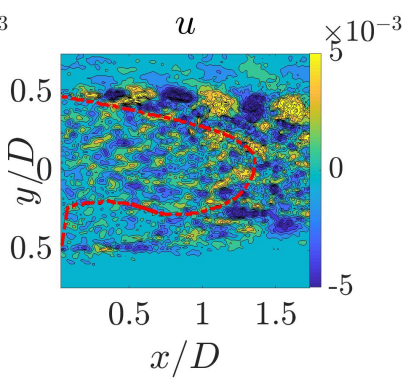

(f)
Figure 7. Representative modes $\phi_{1}^{(5)}$ associated with $\langle S t\rangle \approx 0.2,(\mathrm{a}-\mathrm{b}) ; \phi_{2}^{(5)}$ associated with $\langle S t\rangle \approx 0.04$ (c-d); and $\phi_{3}^{(3)}$ associated with $\langle S t\rangle \approx 1.2$, (e-f). These correspond, respectively, to vortex shedding, bubble pumping and shear layer instability.

in Figure 7 appears to represent the higher-frequency shear layer.

To examine the suitability of the resulting modes for reduced order modelling, the time varying part of the original snapshots of, $(\mathscr{X}-\bar{X})$, were projected on the matrix whose columns are the real and imaginary parts of the three modes discussed above. The power spectral densities of the resulting time series were then obtained using a Thomson multi-taper method with 7 taper functions. Figure 6 shows that the power spectral density for each mode has a peak at a frequency close to the mean frequency of the cluster. The fact that each mode has a dominant frequency can be exploited while constructing a model based on these mode shapes.

\section{Conclusions}

A method of post-processing modes for extracting a small number of dynamically relevant modes was presented. The method groups DMD modes into clusters, using the graph-theoretic notion of maximal cliques, and iter- atively combines similar clusters to form clusters of higher cardinality. Through this iterative process, a sparse representation of the flow can be found which cannot be furtherreduced in dimension. Each cluster is then represented by a single mode. Applying this algorithm to the velocity snapshot data from the immediate wake of a bullet-shaped bluff body of rotation at $R e=1.88 \times 10^{5}$, the most significant modes are found to correspond to vortex shedding and the oscillatory behaviour of the recirculation bubble, consistent with observations in the literature.

\section{REFERENCES}

Baj, P., Bruce, P. J. K. \& Buxton, O. R. H. 2015 The triple decomposition of a fluctuating velocity field in a multiscale flow. Physics of Fluids 27 (7).

Berger, E., Scholz, D. \& Schumm, M. 1990 Coherent vortex structures in thewake of a sphere and a circular disk at rest and under forced vibrations. Journal of Fluids and Structures 4 (3), 231 - 257.

Bjorck, Ake \& Golub, Gene H. 1973 Numerical methods for computing angles between linear subspaces. Mathematics of Computation 27 (123), 579-594.

Bron, Coen \& Kerbosch, Joep 1973 Algorithm 457: Finding all cliques of an undirected graph. Commun. ACM 16 (9), 575-577.

Grilli, Muzio, Schmid, Peter J., Hickel, Stefan \& Adams, Nikolaus A. 2012 Analysis of unsteady behaviour in shockwave turbulent boundary layer interaction. Journal of Fluid Mechanics 700, 16 - 28.

Hemati, Maziar S., Rowley, Clarence W., Deem, Eric A. \& Cattafesta, Louis N. 2017 De-biasing the dynamic mode decomposition for applied koopman spectral analysis of noisy datasets. Theoretical and Computational Fluid Dynamics 31 (4), 349-368.

Jovanovic, Mihailo R., Schmid, Peter J. \& Nichols, Joseph W. 2014 Sparsity-promoting dynamic mode decomposition. Physics of Fluids 26 (2).

Oxlade, Anthony R., Morrison, Jonathan F., Qubain, Ala \& Rigas, Georgios 2015 High-frequency forcing of a turbulent axisymmetric wake. Journal of Fluid Mechanics 770, 305-318.

Rigas, G., Oxlade, A.R., Morgans, A.S. \& Morrison, J.F. 2014 Low-dimensional dynamics of a turbulent axisymmetric wake. Journal of Fluid Mechanics 755.

Schmid, Peter J. 2010 Dynamic mode decomposition of numerical and experimental data. Journal of Fluid Mechanics 656, $5-28$.

Schmid, Peter J. 2011 Application of the dynamic mode decomposition to experimental data. Experiments in Fluids 50 (4), 1123 - 1130.

Tomita, Etsuji, Tanaka, Akira \& Takahashi, Haruhisa 2006 The worst-case time complexity for generating all maximal cliques and computational experiments. Theoretical Computer Science 363 (1), 28 - 42.

Tu, Jonathan H, Rowley, C. W., Luchtenburg, D. M., Brunton, S. L. \& Kutz, J Nathan 2013 On dynamic mode decomposition - theory and applications . 